\title{
Utilizing three years of epidemiological data from medical missions in Cambodia to shape the mobile medical clinic formulary
}

\author{
Jeany Kim Jun ${ }^{a}$, Junia S Koo ${ }^{b}$, Amy Y Kang ${ }^{c}$, Deborah B Chien ${ }^{d}$, Albert Shim ${ }^{e}$, Dale \\ Knutson ${ }^{\mathrm{f}}$, Eda M Kim ${ }^{\mathrm{g}}$
}

\footnotetext{
${ }^{a}$ PharmD, MPH, BCACP, AAP, Assistant Professor of Clinical and Administrative Sciences, Keck Graduate Institute School of Pharmacy, USA

${ }^{\mathrm{b}}$ PharmD, Staff Pharmacist, University of California Los Angeles Medical Center, California, USA

${ }^{\mathrm{c}}$ PharmD, Post-Graduate Year 1 Pharmacy Practice Resident, Department of Clinical Pharmacy, University of California San Francisco School of Pharmacy, USA

${ }^{d}$ PharmD, Per-Diem Pharmacist, Department of Pharmacy, Desert Valley Hospital, California, USA

${ }^{\mathrm{e}} \mathrm{MD}$, Family Medicine and Pediatrics Physician, Department of Internal Medicine and Pediatrics, Cedars Sinai Medical Group, California, USA

${ }^{f} \mathrm{MD}$, Pediatrician, Medical Missionary, Mission to the World, Cambodia

${ }^{\mathrm{g}} \mathrm{MD}$, Family Practice Physician at Mission to the World, USA
}

\begin{abstract}
Objective: The purpose of this project was to gather epidemiological data on common diseases and medications dispensed during medical mission trips to Cambodia to shape the mobile medical clinic formulary.

Methods: Data for patients seen during week-long mobile medical clinics was collected in Cambodia during Septembers 2012 to 2014. Each patient's gender, age, weight, blood pressure, glucose, pertinent laboratory values, diagnoses, and medications dispensed were collected. Blood pressure and glucose levels were measured in patients 18 years and above. Data collected onto paper intake forms were transferred onto spreadsheets without patient identifying information and analyzed for aggregate means, common diseases, and most dispensed medications. This project received institutional review board approval.
\end{abstract}

Results: A total of 1,015 patients were seen over three years. Women made up $61.4 \%$, and the mean age was 41.8 years. The most common diagnosis was gastrointestinal disorders $(22.9 \%)$ that included gastroesophageal reflux disease and intestinal parasites. Next, $20.1 \%$ of patients had hypertension (BP>140/90), 18.0\% had presbyopia, $15.4 \%$ had back and joint pain, followed by $8.8 \%$ with headache, including migraines. Approximately $8.4 \%$ of patients had hyperglycemia (RPG >140 $\mathrm{mg} / \mathrm{dl})$. The top five medications dispensed were acetaminophen, omeprazole, multivitamin, ibuprofen, and metformin. For hypertension, amlodipine and lisinopril were dispensed.

Mar 2017. Christian Journal for Global Health, 4(1): 13-23. 
Conclusion: Cambodia lacks systematic public health collection of epidemiological data for prevalence of diseases. Hence, investigators collected and analyzed information from week-long mobile medical clinics over three years. Proton-pump inhibitors and $\mathrm{H}$. pylori lab tests were recommended for gastrointestinal disorders. Acetaminophen and ibuprofen were recommended for pain. Angiotensin-convertingenzyme inhibitors and dihydropyridine calcium channel blockers were recommended over diuretics since patients were already dehydrated. Metformin was recommended for diabetes. Vitamins and supplements were recommended for malnourished patients. Hemoglobin machines and urine test strips were suggested. This information should help future teams decide what medications and laboratory tests would be the most beneficial for use by medical teams in Cambodia.

\section{Introduction}

Cambodia is located in Southeast Asia with a population of 14.4 million. ${ }^{1}$ Approximately $80.5 \%$ reside in rural areas with 1.3 million people living in the capital city of Phnom Penh. ${ }^{1} \quad$ The life expectancy at birth averages 61 to 64 years for men and women, respectively. ${ }^{2}$ Agriculture with rice production is the country's primary source of revenue. ${ }^{1}$ In addition, small-scale subsistence agriculture serves as another source of revenue; this includes raising fish and livestock. ${ }^{1}$

Around the year 2000, following an era of war and civil conflict, Cambodia entered a decade of rapid economic growth, along with demographic and epidemiological shifts. ${ }^{3}$ Largely from increased tourism and gains in the garment industry, Cambodia's gross domestic product grew at an average annual rate of $7 \%$ in the following decade. ${ }^{4}$ Additionally, the under-5 mortality rate per 1,000 live-births declined from 124 in 2000 to 54 in $2010{ }^{4}$ Even so, Cambodia remains one of the poorest and most underdeveloped countries in Asia, where many are living on a wage of less than $\$ 1.20$ United States Dollar (USD) per day. ${ }^{4}$ About 6,400 deaths occur annually from lack of adequate nutrition. $^{5}$ Malnutrition among the poor leads to stunted growth (reduced height for age) in $40 \%$ of the children and wasting (reduced weight for height) in $11 \%$ of the children. ${ }^{1}$

Despite the many improvements in the health status in Cambodia, great disparities exist between urban and rural areas, and across socioeconomic groups. Rural areas rely heavily on rain for drinking water during the dry season, and less developed sanitation facilities contribute to increased exposure to communicable diseases. ${ }^{1}$ Acute respiratory infection, fever, and diarrhea are common causes of childhood mortality. While many of these conditions are treatable, in a largely fee-based health care system, the cost of healthcare often creates a barrier to those seeking treatment. In addition to communicable diseases, motorvehicular accidents are a major cause of injury or death in urban areas in the age group 20-39. ${ }^{1}$ Nongovernmental organizations (NGOs), sponsored by international nonprofit organizations, bridge the access gap in healthcare through supporting and serving NGOs led by nationals within Cambodia. ${ }^{6}$

\section{Objective}

Mobile medical clinics provide intermittent care in Cambodia to the underserved population. However, in 2011, the lack of available epidemiological data for prevalent diseases or 
medications hampered the preparation of medical teams to provide appropriate treatment for the patients. In order for future medical teams to more accurately secure medications and laboratory tests needed, this project was undertaken. For the purpose of this project, we gathered epidemiological data on the most common diseases and medications dispensed during medical mission teams to Cambodia from 2012 to 2014 in order to shape future mobile medical clinic formularies.

\section{Methods}

This study evaluated all patients seen during three years of week-long mobile medical clinics held in Septembers 2012, 2013, and 2014 in Cambodia. Patients were seen and evaluated by a multidisciplinary team of healthcare professionals from the United States, including physicians, pharmacy students, pharmacists, physical therapists, and registered nurses, working alongside long-term medical missionaries and Cambodian trained health care providers.

Medical mission teams visited Phnom Penh, as well as three rural villages in Kampot Province. In 2012, the medical team spent four days in NeaReay and two days in Phnom Penh, and saw a total of 317 patients. In 2013, the team spent two days in AngKjay, two days in NeaReay, and two days in Phnom Penh and saw a total of 408 patients. In 2014, the team spent one day in Prey Thom, a very rural village without electricity, two days in AngKjay, and two days in NeaReay and saw a total of 290 patients.

Each patient's gender, age, weight, blood pressure, blood glucose, pertinent laboratory values, diagnoses, and dispensed medications were collected. Blood pressure and blood glucose levels were measured in patients 18 years and older. Data were collected onto paper intake forms and then organized into a computerized spreadsheet, without patient identifying information, and stored using a secure web interface.
Descriptive statistics were computed for all study variables. Means for adults 18 years and above were reported. The numbers of medications dispensed and common disease states were identified. This study was approved by the Loma Linda University Institutional Review Board.

\section{Results}

\section{Demographics}

A total of 1,015 patients were included in the data analysis over three years. Women made up $61.4 \%(\mathrm{~N}=624)$, and the mean age was 41.8 years with a range of 2 weeks to 87 years. Infants, children, and teenagers, 0-17 years, included $15.5 \%$ of the patients. Adults, 18-39 years, made up $25.7 \%$, 40-59 years were $37.0 \%$, and 60 years and over included $21.8 \%$ of the sample. In patients 18 years and above $(\mathrm{N}=858)$, the mean weight and height was 56.3 kilograms $(\mathrm{kg})$ and 65.7 inches in men, and $44.9 \mathrm{~kg}$ and 60.7 inches in women. Average body mass index (BMI) was 21.5 and 21.0 $\mathrm{kg} / \mathrm{m}^{2}$ for men and women, respectively.

\section{Common Diseases}

Overall, gastrointestinal (GI) disorders, including gastritis and gastroesophageal reflux disease (GERD), Helicobacter pylori (H. pylori) infection, dyspepsia, and intestinal parasites were the most common disorders diagnosed in $22.9 \%$ of patients of all ages. H. pylori laboratory testing was not available in 2012 and resulted in 27 patients receiving treatment without a laboratory diagnosis. Investigators purchased $H$. pylori testing kits in Cambodia for \$1 USD each. In the subsequent year, only three patients received treatment with a laboratory-confirmed positive test. Seven patients received $H$. pylori eradication treatment in 2014. All patients received albendazole for possible intestinal parasites. Refer to Figure 1 for the top ten most common disorders. 
Figure 1. Top 10 diagnoses in 2012, 2013, and 2014 combined ( $=1015)$

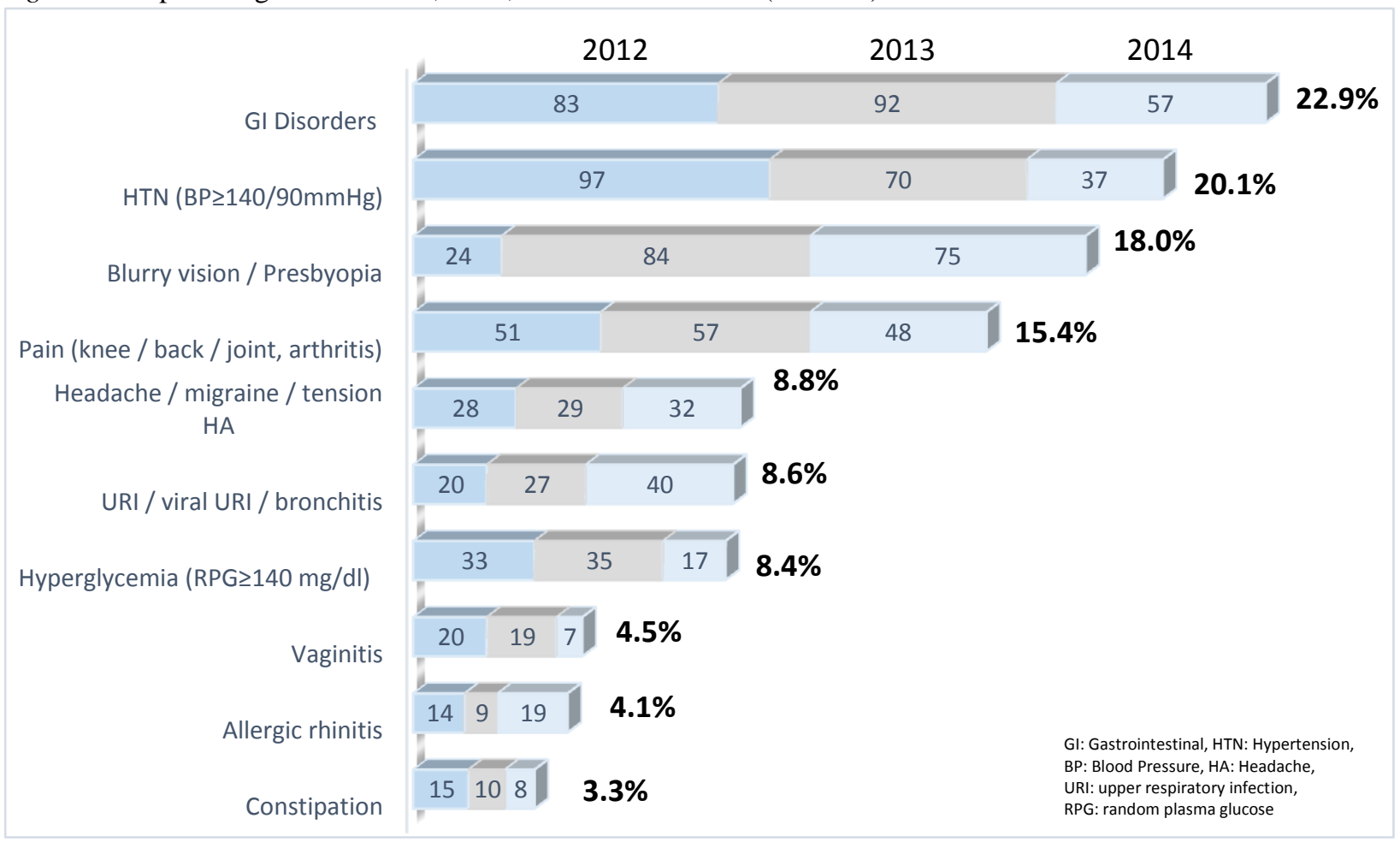

Note: Numbers within figures represent total number of patients with this diagnosis.

Hypertension, defined as a systolic blood pressure of $140 \mathrm{~mm} \mathrm{Hg}$ or above, or diastolic blood pressure of $90 \mathrm{~mm} \mathrm{Hg}$ or above, was the second most common diagnosis. ${ }^{7}$ The prevalence of hypertension among Cambodian adults aged 18 and over was $20.1 \%$ and was similar among men $(21.7 \%)$ and women $(19.7 \%)$. The prevalence of hypertension increased with age, from $9.6 \%$ among those aged $18-39$, to $21.7 \%$ among those $40-59$, to $43.9 \%$ among those 60 and over.

Blurry vision due to apparent presbyopia was a common complaint from patients over 40 years and made up $18 \%$ of the sample. A total of 183 pairs of reading glasses were given over three years. More low-diopter readers $(+1.00$ to +2.00$)$ were dispensed $(\mathrm{N}=139)$ than high-diopter readers $(+2.25$ to +3.50$)(\mathrm{N}=44)$.

Back, knee, and joint pain, including osteoarthritis, was the fourth most common diagnosis at $15.4 \%$. Most of the patients in the rural villages were rice farmers and spent a bulk of their time planting or harvesting rice. Furthermore, head pain, including migraine and tension-type headache, was fourth with $8.8 \%$. Patients with complaints of back and knee pain were referred to the physical therapist (PT). One PT evaluated a total of 113 patients (71 patients in 2013 and 42 patients in 2014) with complaints of back and knee pain, subsequently instructing them in proper exercises and posture.

The overall prevalence of hyperglycemia, defined as a random plasma glucose (RPG) of greater or equal to $140 \mathrm{mg} / \mathrm{dl}$, was $8.4 \%$ and was slightly higher in men (9.6\%) than women $(7.6 \%)$. The prevalence of hyperglycemia increased with age, from $4.2 \%$ among those aged $18-39$, to $11.0 \%$ among those $40-59$, and to $14.8 \%$ among those 60 and over. Severe hyperglycemia, defined as a RPG of $200 \mathrm{mg} / \mathrm{dl}$ or greater, was found in $3.7 \%$ of the patients with similar rates between men $(3.5 \%)$ and women $(3.8 \%)$. The prevalence of severe hyperglycemia increased with age with $0.8 \%$ among 18 - 
39 year olds, $4.8 \%$ among 40-59 year olds, and $5.4 \%$ in those 60 years and over. The average glucose value for the 32 patients with severe hyperglycemia was $355.0 \mathrm{mg} / \mathrm{dl}$.

\section{Common Medications}

The top five medications dispensed were acetaminophen, omeprazole, multivitamin, ibuprofen, and metformin. This paralleled the top diseases to treat gastrointestinal disorders and pain. Since most patients treated for hyperglycemia required the maximum dose of metformin at $2000 \mathrm{mg} / \mathrm{day}$, four tablets of metformin $500 \mathrm{mg}$ were required daily for patients. Most patients with diabetes were given 120 tablets each. Hence, it became one of the top five dispensed medications to treat $8.4 \%$ of the patients. For hypertension, amlodipine and lisinopril were dispensed the most but diuretics were not used much. Most of the hypertension medications were given once daily, and hence, fewer tablets were dispensed. See Table 1 for a list of the top 20 most dispensed drugs during the three years.

Table 1. Top 20 medications dispensed during 2012-2014

\begin{tabular}{lllllc}
\hline Rank & Medications & $\mathbf{2 0 1 2}$ & $\mathbf{2 0 1 3}$ & $\mathbf{2 0 1 4}$ & Total \# tabs \\
\hline 1 & Paracetamol 500 mg (Acetaminophen) & 3880 & 4095 & 4320 & 12295 \\
2 & Omeprazole 20mg & 2606 & 3318 & 1170 & 7094 \\
3 & Multivitamins & 1955 & 1172 & 2670 & 5797 \\
4 & Ibuprofen 200 or 400 mg & 1721 & 1711 & 960 & 4392 \\
5 & Metformin 500 mg or 850 mg & 685 & 1140 & 600 & 2425 \\
6 & Loratadine 10 mg & 310 & 617 & 910 & 1837 \\
7 & Children's vitamin & 0 & 600 & 1200 & 1800 \\
8 & Ferrous fumarate 200 mg or sulfate 325 mg & 687 & 420 & 540 & 1647 \\
9 & Vitamin B12 & 510 & 460 & 300 & 1270 \\
10 & Amlodipine 5 or 10 mg & 400 & 515 & 340 & 1255 \\
11 & Lisinopril 10 mg & 375 & 601 & 210 & 1186 \\
12 & Calcium carbonate 500 mg & 810 & 339 & 0 & 1149 \\
13 & Docusate sodium 100 mg & 434 & 287 & 330 & 1051 \\
14 & Amoxicillin 250 or 500 mg & 310 & 329 & 400 & 1039 \\
15 & Irbesartan 75 mg & 0 & 0 & 990 & 990 \\
16 & Hydrochlorothiazide 25 mg & 900 & 0 & 0 & 900 \\
17 & Aspirin 81 mg & 490 & 270 & 30 & 790 \\
18 & Diphenhydramine 25 mg & 267 & 85 & 270 & 622 \\
19 & Albendazole 400 mg & 256 & 264 & 99 & 619 \\
\hline 20 & Atenolol 50 mg & 540 & 0 & 30 & 570 \\
\hline
\end{tabular}

\section{Discussion}

Epidemiological information guides overseas medical mission medication needs. In 2011, no epidemiological data existed in Cambodia on the prevalence of diseases, especially in the rural areas. The investigators collected and analyzed direct data during week-long mobile medical clinics in the Kampot Province of Cambodia from 2012 to 2014. Public health epidemiological data regarding Cambodia was published in September 2015 by the
World Bank and in 2014 by the World Health Organization $^{4,8-10}$ that includes major causes of death and burden of disease but does not indicate prevalence rates by province. Gastrointestinal complaints were the most common health problems. Included in these complaints were intestinal parasitic infections treated with albendazole 400 mg. Testing kits for H. pylori minimized antibiotics and saved money. Each Cambodian H. pylori antibody test cost $\$ 1$ USD. This test detected antibody presence, which enhanced clinical 
judgment. For \$5 USD, each $H$. pylori sequential therapy eradication packet contained omeprazole 20 $\mathrm{mg}$ twice daily for ten days; amoxicillin $1000 \mathrm{mg}$ twice daily for the first five days; clarithromycin $500 \mathrm{mg}$ twice daily for the next five days; and metronidazole $500 \mathrm{mg}$ twice daily for the next five days. After utilizing $H$. pylori tests, the number of packets given to patients was reduced from 27 in 2012 to 3 in 2013, and 7 in 2014, saving \$75 in two years. All the medications used in the $H$. pylori packet were easily purchased in Cambodia.

A Cambodian survey completed in 2005 showed an unexpectedly high prevalence of hypertension in the relatively poor rural communities. The prevalence of hypertension was $12 \%$ in a rural community (Siem Reap) and $25 \%$ in a semi-urban community (Kampong Cham). ${ }^{11}$ The prevalence of hypertension in this study was in the rang of that of the 2005 survey at $20.1 \%$ in Kampot Province. For reference, the prevalence of HTN in US adults was $29.1 \%$ in $2011-2012 .{ }^{12}$ U.S. trained physicians initially used the Seventh Report of the Joint National Committee (JNC 7) guidelines which recommended beginning patients with diuretics. ${ }^{13}$ However, after viewing urinalysis results with high specific gravity (above 1.030), the clinicians recommended that diuretics not be used first line in the treatment of hypertension. The clinicians, including the pharmacist, recommended other pharmacologic options, like lisinopril or enalapril, an ACE inhibitor, and amlodipine, a dihydropyridine calcium channel blocker. These medications were readily available in Cambodia for patients to purchase at local pharmacies once they ran out of medications received during the mobile clinic visits and inexpensive at $\$ 0.02$ per tablet for lisinopril or enalapril $10 \mathrm{mg}$ and $\$ 0.04$ per tablet for amlodipine $5 \mathrm{mg} .{ }^{14}$

The 2005 survey also reported rates of diabetes in the two provinces. The total prevalence of diabetes was 5\% at Siem Reap and $11 \%$ at Kampong Cham, and the prevalence of impaired glucose tolerance was $10 \%$ at Siem Reap and 15\% at Kampong Cham. Thus, total prevalence of abnormal glucose tolerance was $15 \%$ at Siem Reap and $26 \%$ at Kampong Cham. ${ }^{11}$ However, in 2015, the International Diabetes Federation reported that the prevalence of diabetes in adults in Cambodia aged 20-79 years was only $2.6 \% .{ }^{15}$ According to this study, the prevalence of hyperglycemia, or possibly impaired glucose tolerance, with glucose values between 140 and $199 \mathrm{mg} / \mathrm{dl}$ was $6.2 \%$ with higher rates in men $(8.7 \%)$ than women $(4.7 \%)$. The prevalence of severe hyperglycemia with glucose values $200 \mathrm{mg} / \mathrm{dl}$ and above, which likely indicates diabetes, was $3.7 \%$. Hence, the overall prevalence of hyperglycemia and severe hyperglycemia was $8.4 \%$. In terms of treatment, metformin was the first-line option for diabetes, after establishing baseline renal function through urinalysis tests. Metformin was readily available for purchase in Cambodian pharmacies by the patient once they completed the medications provided during the mobile medical clinic visits. Although inexpensive, sulfonylureas were not recommended due to the risk of hypoglycemia and lack of glucose monitoring by patients. For patients with severe hyperglycemia, insulin treatment was not considered due to limited availability of refrigeration in patients' homes.

Analgesics, such as acetaminophen and ibuprofen, treated back, neck, joint, and headache pain. Based on the needs of the patient population, preparing adequate supplies of these medications is recommended. Interestingly, vaginitis was one of the top ten diagnoses. Limited access to clean water leading to poor hygiene may provide the basis for vaginitis as a common complaint. Antifungal treatments, like fluconazole or antifungal vaginal creams, should be included in the formulary. Loratadine was given for allergic symptoms and mild respiratory complaints with a 10 to 30 day supply. Diphenhydramine was used mostly to treat insomnia with a 14 day supply.

According to the Demographic Health Survey in Cambodia released in 2011, more than 4 in 10 women in Cambodia were anemic, although moderate and severe anemia was relatively rare 
(8\%). In addition, more than half $(55 \%)$ of Cambodian children aged 6-59 months were anemic. $^{16}$ Thus, adult multivitamins (ranked $3^{\text {rd }}$ ) and children's vitamins (ranked $7^{\text {th }}$ ), along with other essential nutrients such as ferrous sulfate $\left(8^{\text {th }}\right)$, vitamin $\mathrm{B} 12\left(9^{\text {th }}\right)$, and calcium carbonate $\left(12^{\text {th }}\right)$, were given in bulk to patients with poor nutritional status. Prenatal vitamins for pregnant and breastfeeding women were given in bulk for at least three to six months to cover the duration of pregnancy or lactation.

Reading glasses, available in low diopters, helped older patients' complaints of blurry vision. Sunglasses were given to protect against cataracts and pterygium. The investigators recommended the H. pylori blood test kit, a hemoglobin machine to test for anemia, and a glucometer. A simple dipstick urinalysis to qualitatively measure urine glucose, protein, leukocytes, and specific gravity helped diagnose infections and understand kidney function.

For chronic diseases such as hypertension and diabetes, it was crucial that the medications selected and started by the short-term mobile medical team be readily available, and not be cost prohibitive for patients to continue treatment once the patients ran out of medications. Hence, mobile teams should consider what medications are available locally when deciding which medications to take with them in the future to treat these diseases.

For sustainability of treatment for patients seen at mobile medical clinics, the short-term medical team was hosted by long-term medical missionaries in partnership with the missionaries located in the rural villages that were visited. After the departure of the short-term team, the long-term medical missionaries went back to these villages bimonthly to bring additional medications and supplies, and provided follow-up care for those who were identified as having a chronic illness such as hypertension and diabetes. Although these results are specific to the rural areas of Cambodia, some aspects may be generalizable to some neighboring South East Asian countries due to the similarities in climate and rural living conditions with limited access to clean water and electricity.

\section{Limitations}

The study faced several limitations during its course. First, this was an observational study with patient information gathered heavily from rural areas, which may limit the generalizability of the study. Second, this study mostly evaluated prevalence at the time of the clinic visit and did not provide information regarding diseases that may have developed after the visit. Third, there was limited availability of diagnostic testing devices which may have limited the physicians' ability to make a definitive diagnosis. Furthermore, patients typically had one blood pressure measurement, whereas at least two are required for a definitive diagnosis. Similarly, only one random blood glucose measurement was done for most patients, so it was difficult to accurately diagnose based on one measurement. With that said, some strategies to address these issues were to return to the same villages bi-monthly to provide follow-up care for those patients who had elevated blood pressures or blood glucose values for a firm diagnosis.

\section{Conclusion}

Delivering cost-effective, high-quality health care services in resource-poor settings remains an enduring challenge. This challenge, however, becomes more formidable for short-term medical mission teams as they are faced not only with limitations in language and culture, diagnostic tools, and treatment options, but also with limitations with respect to local epidemiological data, an indispensable tool for pre-field preparation as well as on-field diagnosis and treatment. The stakes are high given the very poor access to healthcare particularly in rural contexts, the generally high cost of medical mission trips, the limitations of time, and the unavoidable hard decisions the teams must make with respect to what diagnostic tools and medications they will take and which they will 
leave behind. We submit this study not only as a resource for those teams traveling to Cambodia but also elsewhere, where attention to epidemiological data promises to lead to a more thoughtful approach to medical mission trips.

\section{References}

1. National Institute of Statistics, Directorate General for Health, and ICF Macro, 2011. Cambodia demographic and health survey 2010 [Internet]. Phnom Penh, Cambodia and Calverton, Maryland, USA: National Institute of Statistics, Directorate General for Health, and ICF Macro. [cited 2016 January 12]. Available from: https://dhsprogram.com/pubs/pdf/FR249/FR249.pdf

2. Centers for Disease Control and Prevention. Global health - Cambodia [Internet]. [cited 2016 January 12]. Available from: http://www.cdc.gov/globalhealth/countries/cambodia

3. The world factbook. East \& Southeast Asia: Cambodia [Internet]. [cited 2016 January 12]. Available from: https://www.cia.gov/library/publications/the-worldfactbook/geos/cb.html

4. World Bank (2014). World bank databank [Internet]. [cited 2016 January 12]. Available from: http://databank.worldbank.org/.

5. Department of Planning and Health Information. Annex 1 situation analysis [Internet]. [cited 2016 January 12]. Available from: http://www.who.int/health financing/documents/cam frmwrk-annex_1.pdf

6. Meessen B, Van Damme W, Por I, Van Leemput L, Hardeman W. 2002. The new deal in Cambodia: the second year [Internet]. [cited 2016 January 12]. Phnom Penh: MSF Cambodia. Available from: http://www.unicef.org/evaldatabase/files/Cambodia_ New_Deal_rec 347894.pdf

7. James PA, Oparil S, Carter BL, Cushman WC, Dennison-Himmaelfarb C, Handler J, et al. Evidence-based guideline for the management of high blood pressure in adults: report from the panel members appointed to the Eighth Joint National
Committee (JNC 8). JAMA. 2014;311(5):507-20.

Available from: http://dx.doi.org10.1001/jama.2013.284427

8. World Health Organization. Cambodia [Internet]. [cited 2015 September 15.] Available from: http://www.who.int/nmh/countries/khm_en.pdf?ua=1

9. Country statistics and global health estimates by WHO and UN partners [Internet]. [cited 2016 January 26]. Available from: (http://www.who.int/gho/en/) [Last updated: January 2015]

10. Global Burden of Diseases (GBD) Profile: Cambodia [Internet].[cited 2016 January 19] Available from: http://www.healthdata.org/sites/default/files/files/cou ntry_profiles/GBD/ihme_gbd_country_report_camb odia.pdf

11. King H, Lim K, Seng S, Khun T, Roglic G, Pinget M. Diabetes and associated disorders in Cambodia; two epidemiological surveys. Lancet. 2005;366: 1633-9. Available from: http://dx.doi.org/10.1016/S0140-6736(05)67662-3

12. Nwankwo T, Yoon SS, Burt V, Gu Q. Hypertension among adults in the United States: National Health and Nutrition Examination Survey, 2011-2012. NCHS data brief, no 133. Hyattsville, MD: National Center for Health Statistics. 2013.

13. The Seventh Report of the Joint National Committee on Prevention, Detection, Evaluation, and Treatment of High Blood Pressure. NIH Publication No. 045230 August 2004.

14. Internal document for Mercy Medical Center Pharmacy, 2013.

15. International Diabetes Federation. Cambodia 2015 [Internet]. [cited 2016 June 8]. Available from: http://www.idf.org/membership/wp/cambodia

16. National Institute of Statistics, Directorate General for Health, and ICF Macro, 2011. 2010 Cambodia demographic and health survey: key findings [Internet]. [cited 2015 September 29]. Phnom Penh, Cambodia and Calverton, Maryland, USA: National Institute of Statistics, Directorate General for Health, and ICF Macro. Available from: http://dhsprogram.com/pubs/pdf/SR185/SR185.pdf/

Peer Reviewed

Competing Interests: None declared.

Mar 2017. Christian Journal for Global Health, 4(1): 13-23. 
Correspondence: Jeany Kim Jun, Keck Graduate Institute School of Pharmacy, United States. jjun@kgi.edu

Cite this article as: Jun J K, Koo J S, Kang A Y, Chien D B, Shim A, Knutson D, Kim E M. Utilizing three years of epidemiological data from medical missions in Cambodia to shape the mobile medical clinic formulary. Christian Journal for Global Health. Mar 2017; 4(1): 13-23.

(c) Jun J K, Koo J S, Kang A Y, Chien D B, Shim A, Knutson D, Kim E M This is an open-access article distributed under the terms of the Creative Commons Attribution License, which permits unrestricted use, distribution, and reproduction in any medium, provided the original author and source are properly cited. To view a copy of the license, visit https://creativecommons.org/licenses/by/4.0/

www.cjgh.org 